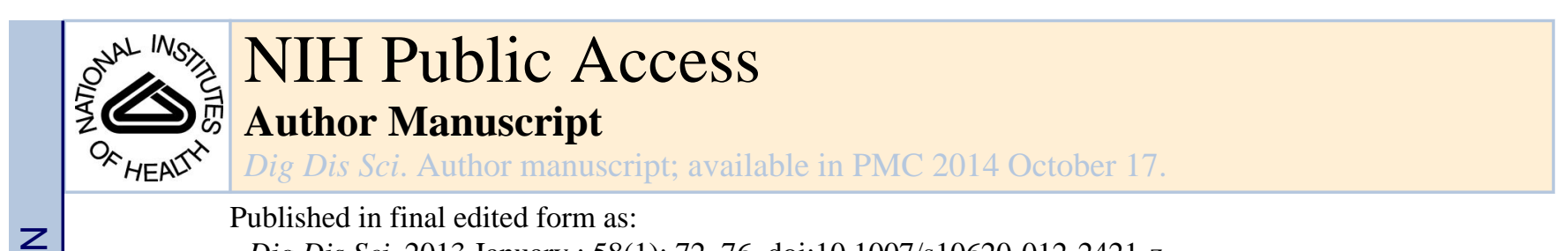

Dig Dis Sci. 2013 January ; 58(1): 72-76. doi:10.1007/s10620-012-2421-z.

\title{
High Fat Diet Differentially Regulates the Expression of Olfactory Receptors in the Duodenum of Obesity-Prone and Obesity-Resistant Rats
}

\author{
Stefany D. Primeaux, \\ Joint Diabetes, Endocrinology and Metabolism Program, Louisiana State University System, \\ Louisiana State University Health Science Center-New Orleans (LSUHSC-NO), New Orleans, LA \\ 70112, USA. Internal Medicine-Endocrinology, Diabetes and Metabolism, Louisiana State \\ University System, Louisiana State University Health Science Center-New Orleans (LSUHSC- \\ NO), 1542 Tulane Ave, Box T4 M-2, New Orleans, LA 70112, USA
}

H. Douglas Braymer, and

Pennington Biomedical Research Center, Baton Rouge LA 70808, USA

George A. Bray

Pennington Biomedical Research Center, Baton Rouge LA 70808, USA

Stefany D. Primeaux: sprime@Isuhsc.edu

\begin{abstract}
Background-The gastrointestinal tract is important in the regulation of food intake, nutrient sensing and nutrient absorption. Obesity-prone Osborne-Mendel (OM) rats are less sensitive to the satiating effects of a duodenal infusion of fatty acids than obesity-resistant S5B/Pl (S5B) rats, suggesting that the gastrointestinal tract differentially senses the presence of fat in these two strains. A microarray analysis was conducted to identify genes that were differentially expressed in the duodenal enterocytes of OM and S5B rats.
\end{abstract}

Aims-The present experiment evaluated the expression of olfactory receptors in the duodenal
enterocytes of OM and S5B rats. It was hypothesized that olfactory receptors present in the
duodenum of OM and S5B rats would be differentially regulated by the intake of a high fat diet.

Methods-The mRNA levels of four olfactory receptors (Olr1744, Olr50, Olr124, Olr1507) were assessed from the duodenal enterocytes of OM and S5B rats consuming a high fat diet for 14 days.

Results-The duodenal mRNA levels of Olr1744, Olr124 and Olr1507 were significantly elevated in OM rats fed the high fat diet, but not S5B rats. No differences in the expression of Olr50 receptor mRNA were detected.

Conclusions-These data suggest that several olfactory receptors present in the duodenum are selectively regulated by high fat diet intake in obesity-prone OM rats. Therefore, these receptors

(C) Springer Science+Business Media New York 2012

Correspondence to: Stefany D. Primeaux, sprimel lsuhsc. edu.

Conflict of interest None. 
may play a role in the sensing and regulation of dietary fat, and may be important for the individual susceptibility to obesity in these two strains.

\section{Keywords}

Obesity-prone; Obesity-resistant; Olfactory receptors; Duodenum; High fat diet

The role of the gastrointestinal tract (GI) in the detection and absorption of nutrients is an increasingly important component for understanding the intake of foods high in fat and the subsequent prevalence of obesity. Following ingestion of nutrients, the release of hormones and the activation of receptors alter the physiology of the GI tract and affect further ingestion of nutrients. Evidence from animal models of diet-induced obesity suggests that the detection of dietary fat by the GI tract is subject to individual differences in susceptibility to obesity. Multiple studies have investigated differential sensing of dietary fat by the GI tract of obesity-prone Osborne-Mendel (OM) and obesity-resistant S5B/Pl (S5B) rats [1-4]. Duodenal infusions of Intralipid (a highly emulsified triglyceride mixture) and sodium linoleate on sham-fed animals suppressed food intake more completely and for a longer time in S5B rats compared to OM rats [1]. Recently, we reported a diminished ability of the GLP-1 agonist, Exendin 4, to reduce high fat diet (HFD) intake in OM rats, compared to S5B rats [4]. These data demonstrated strain differences in the ability of the GI tract to respond to the presence of dietary fat and suggested that the S5B rats were more sensitive to dietary fat than OM rats.

To further investigate the differences in the GI tract of OM and S5B rats, a microarray analysis was conducted from the duodenal enterocytes of OM and S5B rats fed a HFD or a low fat diet (LFD). The results from this microarray analysis established strain differences in response to HFD in genes related to immunity, lipid and fatty acid metabolism, signal transduction and olfaction (see Fig. 1; Table 1) [5]. Though numerous genes were altered in these two rat strains by HFD intake, one gene, Olr1744, was particularly intriguing. The expression of a large number of olfactory receptors contributes to the complexity of the olfactory system's ability to detect and discriminate odorous compounds [6]. Recently, these receptors have been detected in the GI tract $[7,8]$.

Several studies have reported differential protein expression in adipose tissue, liver, and skeletal muscle of obesity-prone and -resistant rats and suggest that these proteins play a role the susceptibility to obesity [9-11]. The purpose of the present experiment was to assess the expression of olfactory receptors in the duodenum of OM and S5B rats consuming a HFD or a LFD. Based on our microarray analysis, it was hypothesized that Olr1744 would be increased by the consumption of HFD in OM rats, but not S5B rats. It was further hypothesized that the expression of several additional olfactory receptors in the duodenum would be differentially affected by the intake of a HFD in OM and S5B rats.

Male OM and S5B rats (8-9 weeks old) were bred in the AAALAC approved Pennington Biomedical Research Center vivarium and housed on a 12/12 h light/dark cycle with food and water available ad libitum. All procedures were approved by the Pennington Center Institutional Animal Care and Use Committee. Animals were given access to a pelleted high fat diet ( $55 \% \mathrm{kcal}$ from fat) or pelleted low fat diet (10\% kcal from fat) for 2 weeks prior to 
sacrifice [4]. Food intake was measured daily and body weight was measured weekly. For real-time PCR, a $2.5-\mathrm{cm}$ section of the duodenum was removed, cleaned and the enterocytes were removed by gentle scraping with a clean metal spatula. Excised duodenal enterocytes were immediately frozen on dry ice and stored at $-80{ }^{\circ} \mathrm{C}$ until further processing.

RNA was isolated from duodenal enterocytes using Tri-Reagent (Molecular Research Ctr, Cincinnati, OH) and RNeasy Minikit procedures (Qiagen, Valencia, CA) and based on previous experiments [12]. A microarray analysis on these samples was conducted by the Pennington Center Genomics Core (see Fig. 1; Table 1). Rigorous requirements for the microarray analysis ( $p$ value of less than 0.05 and fold-change of greater than 4) led to the discovery of a significant alteration in the olfactory receptor, Olr1744, in these strains. The application of slightly less stringent criteria revealed potential diet differences in Olr1507, Olr50, and Olr124. Biological function for these genes was based on the Panther Classification System (www.pantherdb.org).

Reverse transcription was conducted using the High-Capacity cDNA Reverse Transcriptase Kit (Applied Biosystems, Foster City, CA, USA) and based on previous studies [4]. Primers were designed using Primer Express (Applied Biosystems) to assess and verify differences in these four olfactory receptors-Olr1744: For-5'-TCATCTTCTCCTTGCCCTTCTG-3', Rev-5'-AGCACCGGCTGGATCTCA-3'; Olr50: For-5'TTCTATCCTCCTCCACACAACCTAA- ${ }^{\prime}$, Rev-5'-AGGCAGGCATTGTATTCAAT CTC-3'; Olr1507: For-5'-TCATCTTCTCCTTGCCCTTCT G-3', Rev-5'AGCACCGGCTGGATCTCA-3'; Olr124: For-5'-GCCTTGGGCACTTGTGTTG-3', Rev-5'-GCGATGAGTTAGGAAACTGAACAAT-3'; Cyclophilin: For-5'CCCACCGTGTTCTTCGACAT-3', Rev-5'-CTGTCTTTGGAACTTTGTCTGCAA-3' . For real-time PCR, SYBR Green 2× Master Mix (Applied Biosystems), primers and cDNA were added to 384-well plates. The quantity of Olr1744, Olr1507, Olr50, and Olr124 was based on a standard curve and normalized to cyclophilin levels (ABI Prism 7900 Sequence Detection System, Applied Biosystems). A significance level of $p<0.05$ was used for all tests.

Following 14 days access to the HFD or LFD, a significant strain $\times$ diet interaction was detected for body weight gain $(F=20.28, p<0.05$, data not shown). As expected, obesityprone OM rats gained more weight than S5B rats, and OM rats consuming HFD gained more than OM rats consuming LFD. A significant diet $\times$ strain interaction was detected for average daily food intake of HFD and LFD $(F=17.36, p<0.05$, data not shown). Overall, the rats consumed more HFD than LFD, and OM rats consumed more HFD than the S5B rats. A significant diet $\times$ strain interaction was detected for Olr1744 $(F=5.63, p<0.05)$, $\operatorname{Olr1507}(F=5.52, p<0.05)$ and Olr124 $(F=15.47, p<0.05)$ (see Fig. 2a-c). Differences were not detected for Olr50 (see Fig. 2d). Post hoc analyses revealed a significant increase in the duodenal expression of Olr1744, Olr1507 and Olr124 in the duodenal enterocytes of OM rats consuming a HFD, compared to OM rats consuming a LFD and S5B consuming either a HFD or a LFD $(p<0.05)$.

$\mathrm{OM}$ and S5B rats are models used to assess the mechanisms underlying feeding behavior and obesity $[1-4,13,14]$. OM rats are susceptible to diet-induced obesity and gain more 
weight and body fat when eating a HFD than S5B rats, which are resistant to diet-induced obesity. The purpose of the present experiment was to examine HFD-induced differences in the expression of several olfactory receptors in the duodenal enterocytes of OM and S5B rats. Based on a microarray analysis, it was hypothesized that Olr1744 would be increased by the consumption of HFD in OM rats, but not S5B rats. Several additional olfactory receptors were investigated.

The criteria to detect gene expression differences in this microarray analysis were rigorous and led to the detection of six genes down-regulated by HFD in OM rats, ten genes downregulated by HFD in S5B rats, ten genes up-regulated by HFD in OM rats and five genes upregulated by HFD in S5B rats (see Table 1; Fig. 1). An effect of diet was detected in the olfactory receptor, Olr1744 [5]. The present analysis was expanded to include olfactory receptors which showed differential expression but did not reach statistical significance $(p>$ 0.05). Expression of Olr1744, Olr1507, and Olr124 mRNA in the duodenal enterocytes was significantly elevated in the obesity-prone OM rats consuming a HFD (See Fig. 2).

However, expression of Olr50 mRNA in the duodenal enterocytes was not altered by strain or diet, suggesting that only specific olfactory receptors are modulated by the intake of a HFD.

Previous investigations examining the potential function of olfactory receptors in the GI tract revealed a role for these receptors in the release of serotonin from the GI tract [7, 8]. The expression of olfactory receptors in the human GI tract has been evaluated and activation of olfactory receptors in these cells has been linked to increased influx and increased intracellular levels of $\mathrm{Ca}++$ and increased release of serotonin [7, 8]. Serotonin controls gut motility and secretion, enteric nervous activity and has been implicated in pathologic conditions such as irritable bowel syndrome, eating disorders, vomiting and diarrhea [7, 8, 15-22]. Serotonin receptor type 3 appears to play a prominent role in GIrelated pathology. In people, variants in the serotonin receptor types $3 \mathrm{~A}$ and $3 \mathrm{~B}$ have been associated with anorexia nervosa and bulimia nervosa [19, 22]. Additionally, serotonin receptor type 3 antagonists are used to treat chemotherapy-induced nausea and vomiting and irritable bowel syndrome [21, 22].

In the OM and S5B rats, the differential expression of olfactory receptors in the duodenal enterocytes by HFD may affect the release of serotonin in the GI tract. Earlier studies from this laboratory have investigated the role of serotonin on the intake of HFD in OM and S5B rats $[23,24]$ and demonstrated strain differences in the response to serotonergic drugs. Overall these data report a significant increase in mRNA levels of several olfactory receptors in the duodenum of obesity-prone OM rats fed a HFD. Olfactory receptors are chemoreceptors and though their exact role in the GI tract is not understood, it is apparent from these data that these receptors are susceptible to diet manipulations. More studies are needed to examine the function of these olfactory receptors in the detection of fat/fatty acids and their potential modulation of serotonin and GI motility in these animal models.

\section{Acknowledgments}

This research was supported by NIDDK 32089 to G. A. Bray. The authors would like to thank Eli Bench, Christine Blackmon, Katherine Pyburn and Raphael Malbrue for their assistance on this project. This work was supported in 
part by NINDS49177 to G.M. Holmes, P20-RR021945 from the National Center for Research Resources and NIH NORC (CNRU) Center Grant 1P30 DK072476.

\section{References}

1. Greenberg D, McCaffery J, Potack JZ, Bray GA, York DA. Differential satiating effects of fats in the small intestine of obesity-resistant and obesity-prone rats. Physiol Behav. 1999; 66:621-626. [PubMed: 10386906]

2. Liu X, York DA, Bray GA. Regulation of ghrelin gene expression in stomach and feeding response to a ghrelin analogue in two strains of rats. Peptides. 2004; 25:2171-2177. [PubMed: 15572207]

3. Pittman D, Smith KR, Crawley ME, et al. Orosensory detection of fatty acids by obesity-prone and obesity-resistant rats: strain and sex differences. Chem Sens. 2008; 33:449-460.

4. Primeaux SD, Barnes MJ, Braymer HD, Bray GA. Sensitivity to the satiating effects of Exendin 4 is decreased in obesity-prone Osborne-Mendel rats compared to obesity-resistant S5B/Pl rats. Int J Obes. 2010; 34:1427-1433.

5. Primeaux SD, Braymer DH, Bray GA. High fat diet differentially induces genes for fatty acid metabolism, immunity, olfaction and signal transduction in enterocytes from obesity-prone and obesity-resistant rats. Obes Rev. 2010; 11:91.

6. Fleischer J, Breer H, Strotmann J. Mammalian olfactory receptors. Front Cell Neurosci. 2009:3. [PubMed: 19562086]

7. Braun T, Voland P, Kunz L, Prinz C, Gratzl M. Enterochromaffin cells of the human gut: sensors for spices and odorants. Gastroenterology. 2007; 132:1890-1901. [PubMed: 17484882]

8. Kidd M, Modlin IM, Gustafsson BI, Drozdov I, Hauso O, Pfragner R. Luminal regulation of normal and neoplastic human EC cell serotonin release is mediated by bile salts, amines, tastants, and olfactants. Am J Physiol Neuroreg Mot. 2008; 295:G260-G272.

9. Joo JI, Yun JW. Gene expression profiling of adipose tissues in obesity susceptible and resistant rats under a high fat diet. Cell Physiol Biochem. 2011; 27:327-340. [PubMed: 21471722]

10. Kim DH, Choi JW, Joo JI, et al. Changes in expression of skeletal muscle proteins between obesity-prone and obesity-resistant rats induced by a high fat diet. J Proteome Res. 2011; 10:12811292. [PubMed: 21142077]

11. Wang X, Choi JW, Joo JI, et al. Differential expression of liver proteins between obesity-prone and obesity-resistant rats in response to high fat diet. Br J Nutr. 2011; 106:612-626. [PubMed: 21535901]

12. Primeaux SD, Blackmon C, Barnes MJ, Braymer HD, Bray GA. Central administration of the RFamide peptides, QRFP-26 and QRFP-43, increases high fat food intake in rats. Peptides. 2008; 29:1994-2000. [PubMed: 18765262]

13. Barnes MJ, Holmes G, Primeaux SD, York DA, Bray GA. Increased expression of mu opioid receptors in animals susceptible to diet-induced obesity. Peptides. 2006; 27:3292-3298. [PubMed: 16996647]

14. Primeaux SD, Barnes MJ, Bray GA. Olfactory bulbectomy increases food intake and hypothalamic neuropeptide Y in obesity-prone, but not obesity-resistant rats. Behav Brain Res. 2007; 180:190196. [PubMed: 17420059]

15. Cirillo C, Vanden Berghe P, Tack J. Role of serotonin in gastrointestinal physiology and pathology. Minerva Endocrinol. 2011; 36:311-324. [PubMed: 22322654]

16. Gershon MD. Review article: serotonin receptors and transports-roles in normal and abnormal gastrointestinal motility. Ailment Pharmcol Ther. 2004; 20:3-14.

17. Gershon MD, Liu MT. Serotonin and neuroprptoection in functional bowel disorders. Neurogastroenterol Motil. 2007; 19:19-24. [PubMed: 17620084]

18. Gorard DA, Libby GW, Farthing MJ. 5-Hydroxytryptamine and human small intestinal motility: effect of inhibiting 5-hydroxy-tryptamine reuptake. Gut. 1994; 35:496-500. [PubMed: 8174987]

19. Hammer C, Kapeller J, Endele M, et al. Functional variants of the serotonin receptor type $3 \mathrm{~A}$ and B gene associated with eating disorders. Pharmacogenet Genomics. 2009; 19:790-799. [PubMed: 19741568]

Dig Dis Sci. Author manuscript; available in PMC 2014 October 17. 
20. Machu TK. Therapeutics of 5-HT3 receptor antagonists: current uses and future directions. Pharmac Ther. 2011; 130:338-347.

21. Niesler B, Kapeller J, Hammer C, Rappold G. Serotonin type 3 receptor genes: hTR3A, B, C, D, E. Pharmacogenomics. 2008; 9:501-504. [PubMed: 18466097]

22. Walstab J, Hammer C, Bonisch H, Rappold G, Niesler B. Naturally occurring variants in the HTR3B gene significantly alter properties of human heteromeric 5-hydroxytryptamine-3A/B receptors. Pharmacogenet Genomics. 2008; 18:793-802. [PubMed: 18698232]

23. White CL, Ishihara Y, York DA, Bray GA. Effect of meta-chlorophenylpiperazine and cholecystokinin on food intake of Osborne-Mendel and S5B/P1 rats. Obesity. 2007; 15:624-631. [PubMed: 17372312]

24. White CL, Kashima K, Bray GA, York DA. Effect of serotonin 1-A agonist on food intake of Osborne-Mendel and S5B/P1 rats. Physiol Behav. 2000; 68:715-722. [PubMed: 10764902] 
A
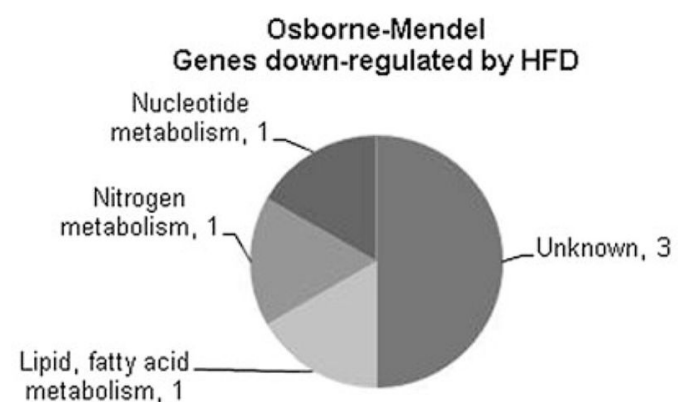

B

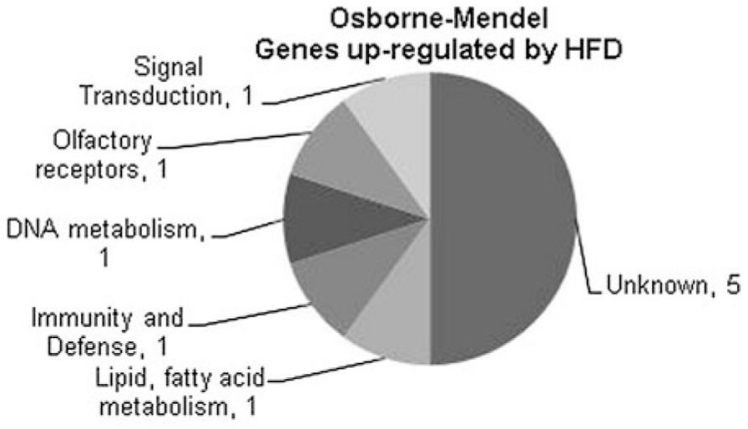

C

S5BIPI

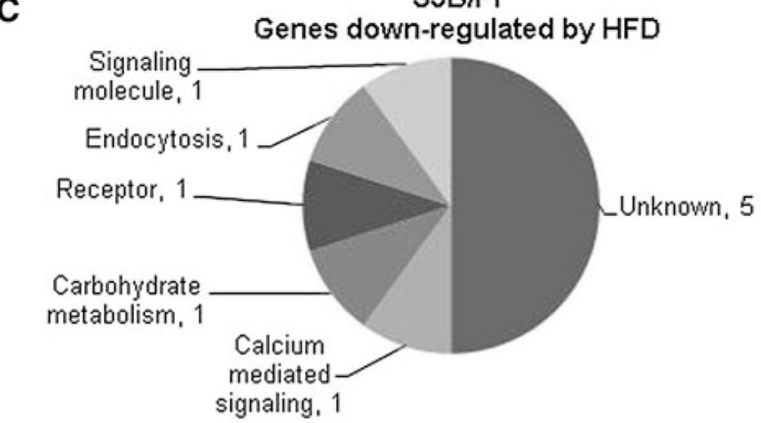

D

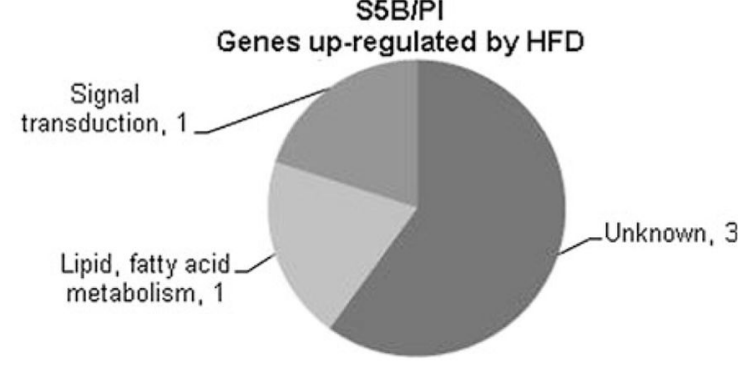

Fig. 1.

A microarray analysis was conducted on duodenal enterocytes Osborne-Mendel (OM) and S5B/Pl (S5B) rats fed a high fat diet (HFD) or low fat diet (LFD) for 14 days. Biological function for these genes was based on the Panther Classification System. a Genes downregulated by HFD in obesity-prone OM rats. b Genes up-regulated by HFD in obesity-prone OM rats. $\mathbf{c}$ Genes down-regulated by HFD in obesity-resistant S5B rats. d Genes upregulated by HFD in obesity-resistant rats. $p<0.05$ and fold-change greater than 4 

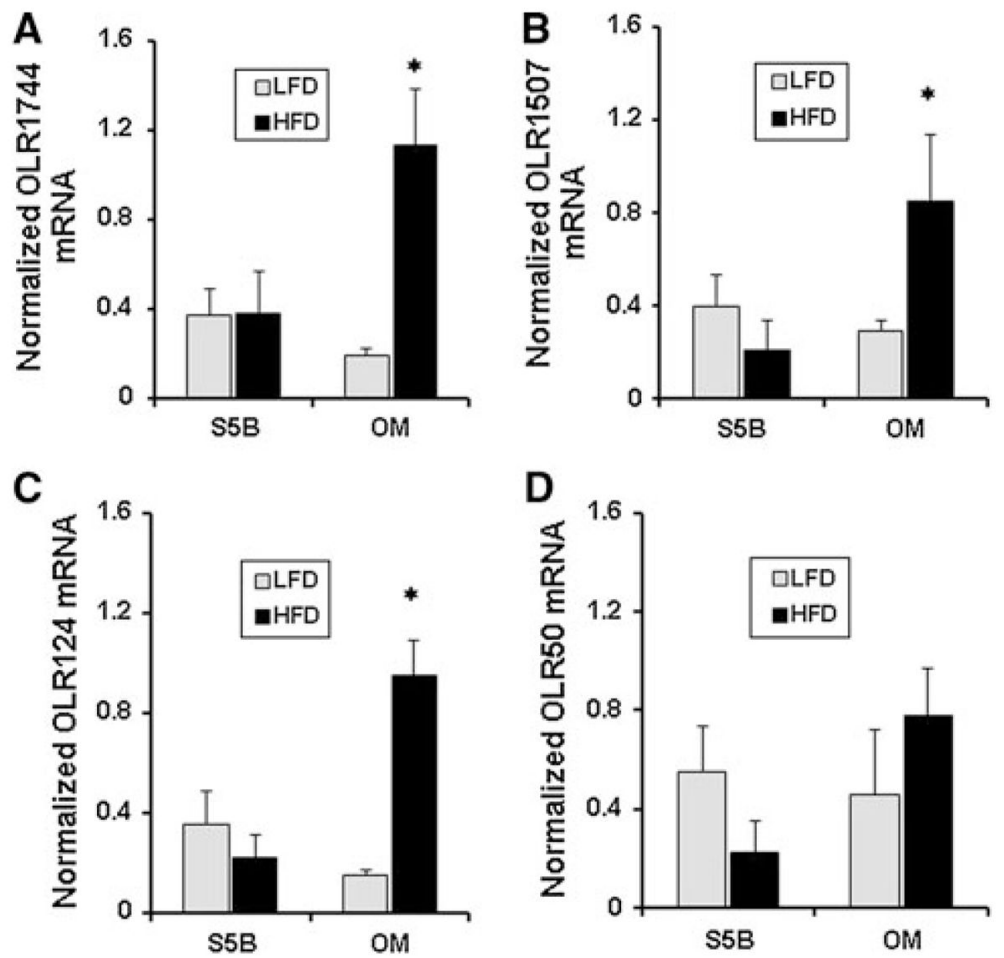

Fig. 2.

Olfactory receptor mRNA levels were measured in the duodenal enterocytes of OsborneMendel (OM) and S5B/Pl (S5B) rats consuming a high fat diet (HFD) or a low fat diet (LFD) for 14 days. a The olfactory receptor, Olr1744, mRNA was increased in the duodenal enterocytes following the consumption of the HFD in obesity-prone OM rats. $\mathbf{b}$ The olfactory receptor, Olr1507, mRNA was increased in the duodenal enterocytes of OM rats consuming the HFD. c The olfactory receptor, Olr124, mRNA was increased in the enterocytes of obesity-prone OM rats consuming a high fat diet. $\mathbf{d}$ The olfactory receptor, Olr50, mRNA was not altered by the consumption of a high fat diet in OM or S5B rats. Data are shown as mean \pm SEM, ${ }^{*} p<0.05,(n=6-9$ rats/group $)$ 


\section{Table 1}

Genes altered by the consumption of a high fat diet in obesity-prone and obesity-resistant rats

\begin{tabular}{|c|c|c|c|}
\hline Gene & Gene ID $^{a}$ & Fold change & $p$ Value \\
\hline \multicolumn{4}{|c|}{ Down-regulated by high fat diet in OM rats (LFD/HFD) } \\
\hline \multicolumn{4}{|l|}{ Lipid, fatty acid metabolism } \\
\hline Cytochrome P450, subfamily $1 \mathrm{~A}$ & Cyp1a1 & 10.52 & $p=0.048$ \\
\hline \multicolumn{4}{|l|}{ Nitrogen metabolism } \\
\hline Nitric oxide synthase & Nos & 4.53 & $p=0.001$ \\
\hline \multicolumn{4}{|l|}{ Nucleotide metabolism } \\
\hline Homeobox protein distal-less DLX & Dlx3 & 4.09 & $p=0.026$ \\
\hline \multicolumn{4}{|c|}{ Up-regulated by high fat diet in $O M$ rats $(H F D / L F D)$} \\
\hline \multicolumn{4}{|l|}{ DNA metabolism } \\
\hline A Helicase Reco5 & Recq15 & 4.0 & $p=0.004$ \\
\hline \multicolumn{4}{|l|}{ Immunity and defense } \\
\hline Fibrinogen alpha/alpha E chain & Fga & 4.98 & $p=0.002$ \\
\hline \multicolumn{4}{|l|}{ Lipid, fatty acid metabolism } \\
\hline Apolipoprotein C-II & Apoc2 & 5.59 & $p=0.034$ \\
\hline \multicolumn{4}{|l|}{ Olfactory receptors } \\
\hline Olfactory receptor MOR263 & Olr1744 & 5.10 & $p=0.013$ \\
\hline \multicolumn{4}{|l|}{ Signal transduction } \\
\hline Angiopoietine-1 & Angpt1 & 4.01 & $p=0.019$ \\
\hline \multicolumn{4}{|c|}{ Down-regulated by high fat diet in $S 5 B$ rats ( $L F D / H F D)$} \\
\hline \multicolumn{4}{|l|}{ Calcium mediated signaling } \\
\hline Calcium binding protein & $\mathrm{L}-\mathrm{CaBP} 2$ & 4.89 & $p=0.001$ \\
\hline \multicolumn{4}{|l|}{ Carbohydrate metabolism } \\
\hline Solute carrier family 2 & Slc2a5 & 8.21 & $p=0.0004$ \\
\hline \multicolumn{4}{|l|}{ Receptors } \\
\hline Acetylcholine receptor, nicotinic $\beta 1$ & Chrnb1 & 6.49 & $p=0.012$ \\
\hline $\mathrm{P} 2 \mathrm{Y}$ purinoreceptor 12 & $\mathrm{P} 2 \mathrm{y} 12$ & 5.24 & $p=0.019$ \\
\hline Latrophilin 3 & Lphn3 & 4.82 & $p=0.044$ \\
\hline \multicolumn{4}{|l|}{ Endocytosis } \\
\hline Vacuolar membrane protein & Vps11 & 4.02 & $p=0.036$ \\
\hline \multicolumn{4}{|l|}{ Signaling molecule } \\
\hline DAPP1/BAM32 & Dapp1 & 4.49 & $p=0.035$ \\
\hline \multicolumn{4}{|c|}{ Up-regulated by high fat diet in $S 5 B$ rats $(H F D / L F D)$} \\
\hline \multicolumn{4}{|l|}{ Lipid, fatty acid metabolism } \\
\hline 2,4 dienoyl-CoA reductase & Decr1 & 6.29 & $p=0.35$ \\
\hline \multicolumn{4}{|l|}{ Signal transduction } \\
\hline RAS homolog gene family, member V & Rhov & 4.21 & $p=0.036$ \\
\hline
\end{tabular}

Dig Dis Sci. Author manuscript; available in PMC 2014 October 17. 